\title{
ELECTROWEAK PHYSICS AND PHYSICS BEYOND THE STANDARD MODEL
}

\author{
L. BELLAGAMBA \\ INFN Bologna, \\ Via Irnerio 46, I-40126 Bologna, Italy, \\ E-mail: Lorenzo.Bellagamba@bo.infn.it \\ E. SAUVAN \\ CPPM, IN2P3-CNRS et Université de la Méditerranée, \\ 163 av. de Luminy F-13288 Marseille, France, \\ E-mail: sauvan@cppm.in2p3.fr \\ H. SPIESBERGER \\ Johannes-Gutenberg-Universität Mainz, \\ Staudinger Weg 7, D-55099 Mainz, \\ E-mail: hspiesb@thep.physik.uni-mainz.de
}

\begin{abstract}
We summarize the recent results on electroweak physics and physics beyond the Standard Model that have been presented at the XIV International Workshop on Deep Inelastic Scattering 2006.
\end{abstract}

\section{Top Mass and Electroweak Precision Measurements}

A precise measurement of the top mass is a central goal of the CDF and D0 experiments which, by 2006, collected an integrated luminosity of $\sim 1 \mathrm{fb}^{-1}$ during the Tevatron run II operations. The top mass enters as an important parameter in the calculation of electroweak (EW) observables. In global fits of the EW observables to Standard Model (SM) parameters, the top mass therefore provides an indirect constraint on the Higgs mass and therefore plays a crucial role in the understanding of the SM Higgs sector. It is hence highly desirable to improve the precision of the top mass measurement in order to reach a precision comparable to that of the other relevant EW parameters, typically of the order $0.1 \%-1 \%$. Figure 1 which shows a compilation of measurements from both D0 and CDF, witnesses the great progress obtained in the last few years due to the increased statistics, the 


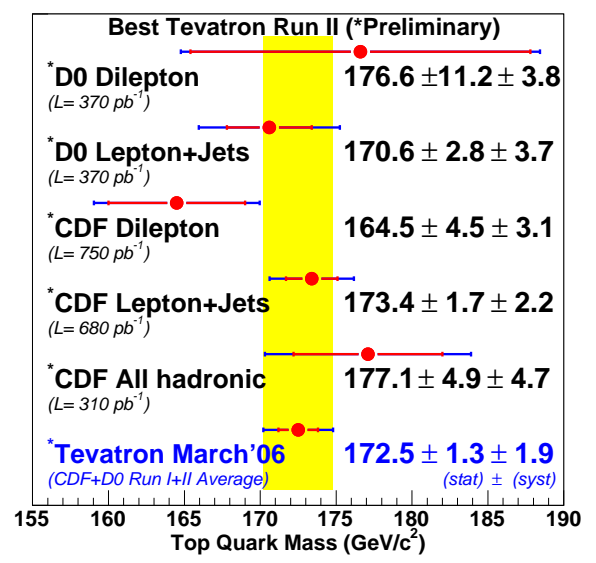

Figure 1. Compilation of top mass measurements at the Tevatron.

upgrade of the detectors for run II and the use of new analysis techniques which allow to better control the main systematic uncertainties.

The systematic error is dominated by the uncertainty on the jet energy scale (JES). Important progress in reducing the JES uncertainty has been obtained using the template and the matrix element methods ${ }^{1}$. The most precise measurement comes from the lepton + jet channel which gives the best compromise between statistics and background. The di-lepton channel gives important contribution since it is less affected by the JES while the all-hadronic channel suffers from a large background and its contribution to the top mass measurement is, at the moment, negligible. The combination of the different measurements gives $M_{\text {top }}=172.5 \pm 1.3$ (stat) \pm 1.9 (sys) GeV. In the coming years of data taking with $4-8 \mathrm{fb}^{-1}$ of integrated luminosity foreseen up to the end of operations at the Tevatron, the di-lepton channel is expected to become dominated by systematic errors and also the allhadronic channel will contribute significantly, allowing to reach a top mass precision of $\sim 1.5 \mathrm{GeV}$.

The Tevatron measurements will provide the most precise top mass value also during the first years of data taking at the LHC. Top production at the LHC experiments will play an important role in the detector commissioning, since it produces a rich topology with $b$-jets, missing $P_{T}$, high- $P_{T}$ jets and high- $P_{T}$ leptons. This involves several detection aspects allowing a global monitoring of the detector performances ${ }^{2}$. A precision of the order of or better than the one at the Tevatron could be reached with 


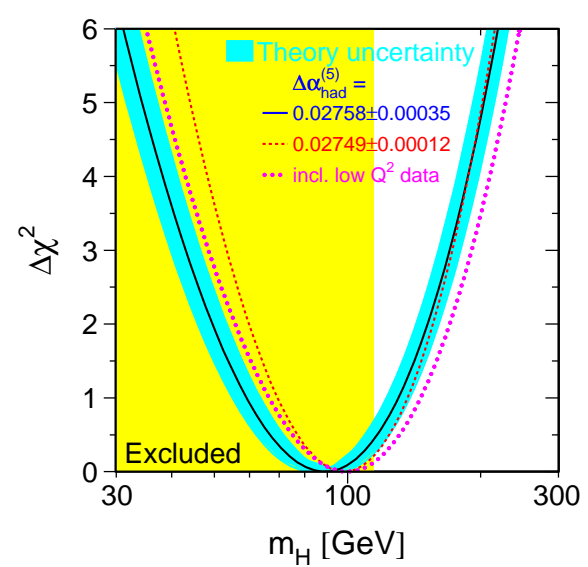

Figure 2. Higgs mass dependence of the $\chi^{2}$ of the electroweak parameter fit.

$10 \mathrm{fb}^{-1}$ of integrated luminosity.

The new Tevatron measurement of the top mass can be used, together with other precise electroweak measurements, for a global fit which allows to extract the SM parameters and test the compatibility of the theory with the present experimental picture. Since many of the EW observables are sensitive to the top mass via radiative corrections, the Higgs mass resulting from these global fits is affected by the top mass. Figure 2 shows the $\chi^{2}$ of the electroweak fit as a function of the Higgs mass. The experimental results clearly favor a light Higgs; masses above $175 \mathrm{GeV}$ (207 GeV taking into account the LEP2 limit) are excluded at 95\% CL.

Also the HERA experiments, exploiting polarized electron and positron beams, have started to contribute to electroweak precision measurements. In particular a combined electroweak and QCD fit ${ }^{3}$ allows to extract the EW neutral current (NC) vector and axial vector couplings of the $Z$ boson to quarks. Figure 3 shows the ZEUS constraints in the plane of the vector $\left(v_{u}, v_{d}\right)$ and axial vector $\left(a_{u}, a_{d}\right)$ couplings. The results are in agreement with the SM expectations and competitive with or better than the Tevatron and LEP constraints.

\section{Higgs Searches}

In the next few years, with the increasing integrated luminosity delivered by the Tevatron and the start-up of the LHC, the range of mass values where the SM Higgs is expected will be accurately scanned. At the Tevatron the Higgs ${ }^{4}$ can be singly produced via gluon-gluon $(g g)$ fusion or in association 

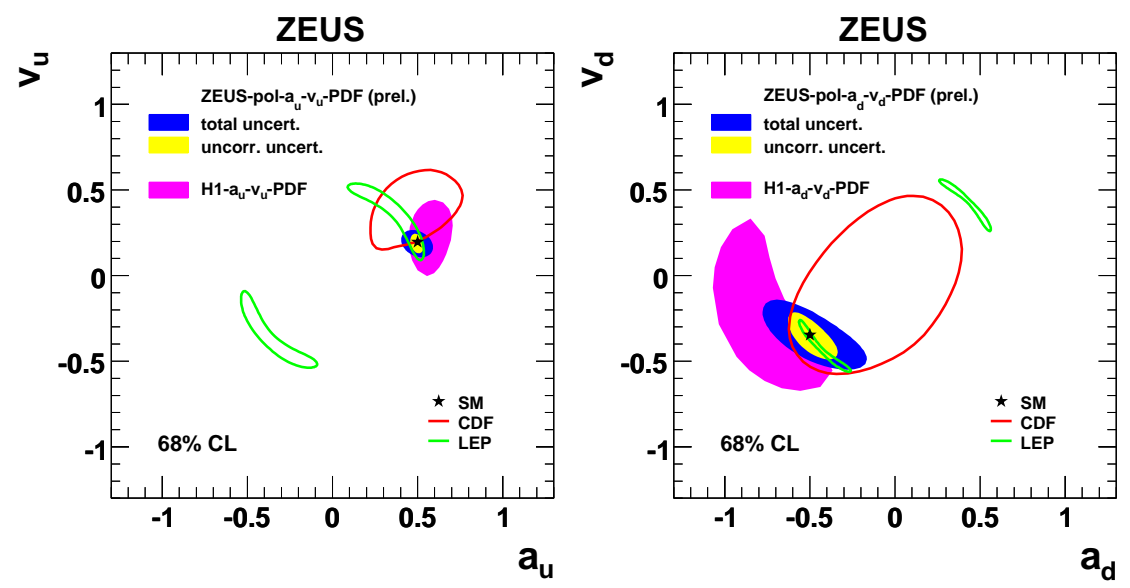

Figure 3. ZEUS constraints for EW NC vector and axial vector couplings of the $Z$ boson to quarks.

with a $W$ or a $Z$ boson in $q \bar{q}$ annihilation. The $g g$ fusion is typically a factor of 5 larger, but for a light Higgs $\left(M_{H} \lesssim 135 \mathrm{GeV}\right)$, when $H \rightarrow$ $b \bar{b}$ is the dominant decay, the Higgs signal is overwhelmed by the QCD background. In this case the associate production with a weak boson allows to control the background requiring high- $P_{T}$ leptons and/or missing $P_{T}$. If the Higgs is heavier, the decay into a couple of weak bosons becomes dominant and single Higgs production via $g g$ fusion is the most sensitive channel. All this is illustrated in Figure 4 where the present 95\% CL limits from $\mathrm{CDF}$ and $\mathrm{D} 0$ are compared with the SM expectations. At the moment, for $M_{\mathrm{H}}=115 \mathrm{GeV}$, i.e. just above the LEP2 exclusion limit, the sensitivity is $\sim 15$ times above the SM expectation. At least $2 \mathrm{fb}^{-1}$ are needed to start excluding part of the mass range and an integrated luminosity of $8 \mathrm{fb}^{-1}$ will allow to cover all the mass range up to $180 \mathrm{GeV}$.

Also the LHC experiments, Atlas and CMS, will participate in the competition for a possible Higgs discovery ${ }^{5}$ in the first years of data taking. In any case, at the LHC the experimental conditions are particularly demanding for a light Higgs just above the LEP2 limit. In this case the most relevant channels are $H \rightarrow \gamma \gamma$ and the associated production of the Higgs with a top pair $(H t \bar{t}, H \rightarrow b \bar{b})$. A corresponding analysis requires a very accurate detector calibration which will be difficult to reach in the early data taking period. The signal detection will be significantly less challenging for larger Higgs masses. Figure 5 shows the CMS perspectives for a possi- 


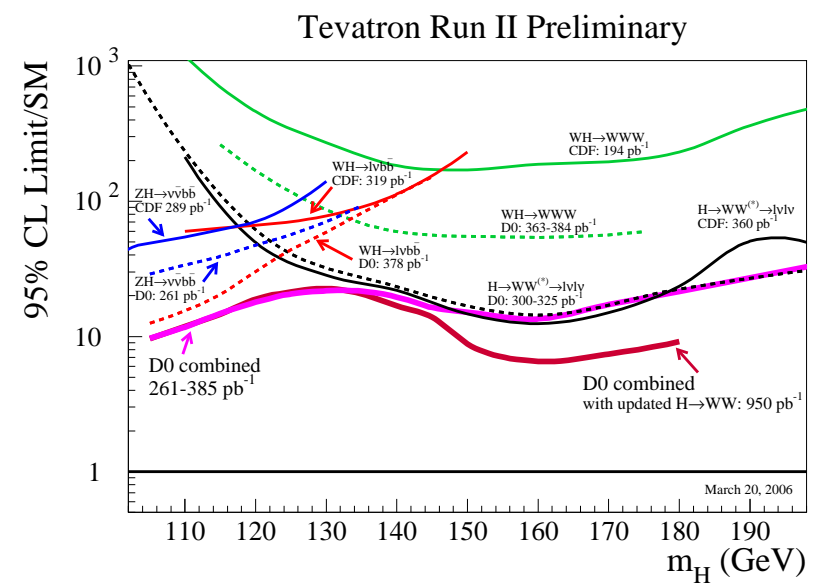

Figure 4. Comparison of the Higgs production limits from CDF and D0 with the SM expectations.

ble discovery as a function of the Higgs mass and for different integrated luminosities. This picture shows that one may expect strong competition between the Tevatron and LHC experiments at the end of 2009 .

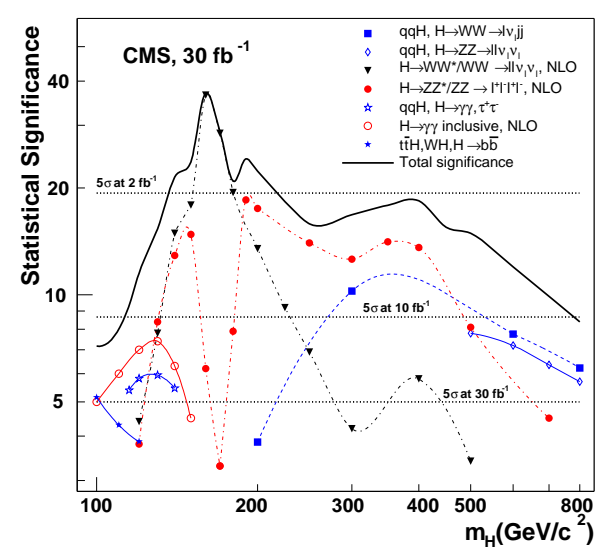

Figure 5. CMS perspectives for a possible Higgs discovery as a function of the mass and for different integrated luminosities. 


\section{Top and Weak Boson Production}

Recently, important progress has been made also in the precision of measurements of the top production cross section ${ }^{6}$. The most precise measurements come from the lepton+jet channel; but, as illustrated in Figure 6 showing a compilation of CDF results, also the other channels give a sizable contribution. The CDF combined result is $\sigma_{t}=7.3 \pm 0.5$ (stat) \pm 0.6 (sys) \pm 0.4 (lumi) pb, $15 \%$ better than the most precise single measurement. The results are in good agreement with theoretical calculations at NLO (also shown in the figure). The D0 collaboration obtained comparable results.

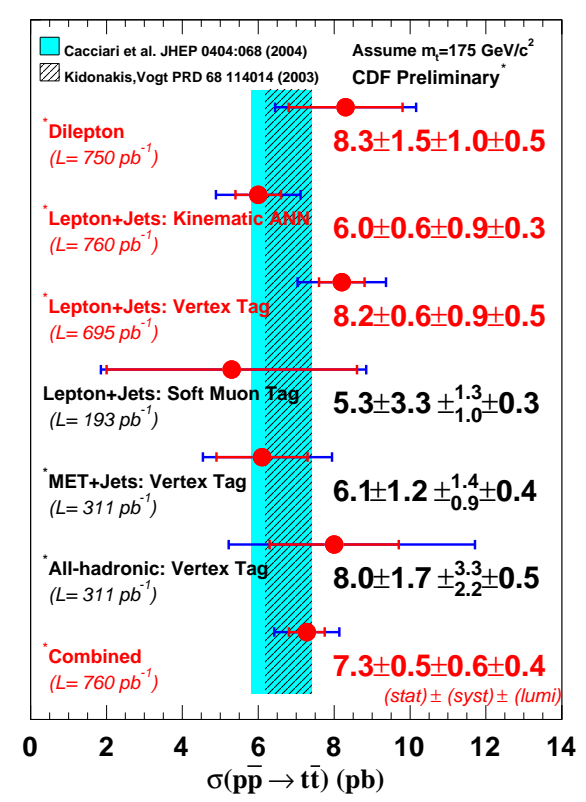

Figure 6. Compilation of measurements of the top production cross section at the Tevatron by the CDF Collaboration. Theoretical predictions based on NLO calculations are also shown.

Single production of electroweak bosons represents a benchmark analysis for D0 and CDF; assuming the validity of SM predictions for its cross section, it can be used to measure or cross check the luminosity. This procedure can be particularly useful at the LHC. Both for single and diboson production ${ }^{7}$ studied in the leptonic decay channels, the agreement between experimental and theoretical results is remarkable. Figure 7 shows 


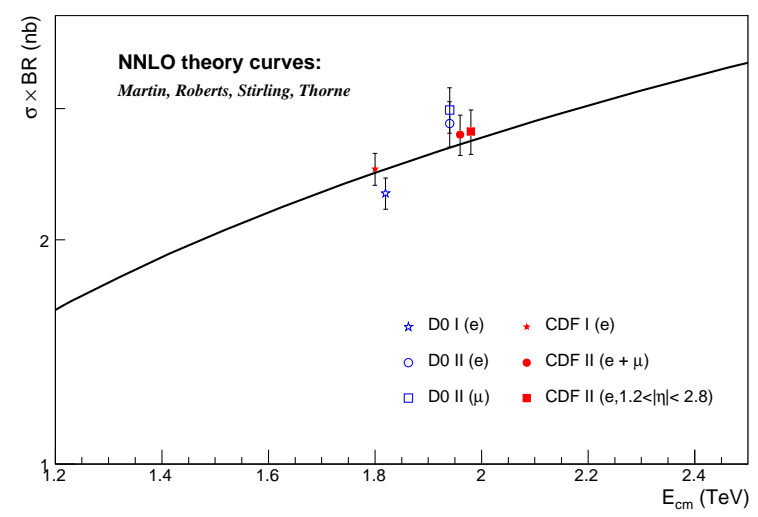

Figure 7. $W$ production cross sections measured by CDF and D0. The measurements obtained both at Tevatron I and II agree well with NNLO predictions also shown in the plots. The measurements at two different center of mass energies have been displaced for clarity.

such good agreement for $W$ production at the Tevatron, both for runs I and II.

A crucial prerequisite for these analyses is the reliability of predictions from QCD calculations including higher-order corrections. Calculations at NLO are standard nowadays. Recent studies of $W$ boson production at large transverse momentum have shown that soft-gluon corrections at NNLO can be important for a reduction of the residual scale dependence ${ }^{8}$. Their inclusion is therefore essential to obtain predictions with reduced theoretical uncertainties.

\section{Flavor Physics at the B-Factories}

The B-factories at SLAC and KEK are performing very well, continuously increasing statistics. At the moment, the integrated luminosity is $\sim 560 \mathrm{fb}^{-1}$ for the Belle collaboration at KEK and $\sim 330 \mathrm{fb}^{-1}$ for the BaBar collaboration at SLAC. Both experiments make significant progress in studies of $\mathrm{CP}$ violation and rare decays. The recent precision measurements of the sides ${ }^{9}$ and the angles ${ }^{10}$ of the unitarity triangle have improved the constraints in the $\rho-\eta$ plane, the two least known parameters of the Cabibbo-Kobayashi-Maskawa matrix (see Figure 8). Both Babar and Belle have also studied a large number of rare B-decays ${ }^{11}$, as well as lepton-flavorviolating and rare decays ${ }^{12}$ of the $\tau$ lepton, observing no deviations from SM predictions and setting limits on new physics which are competitive 


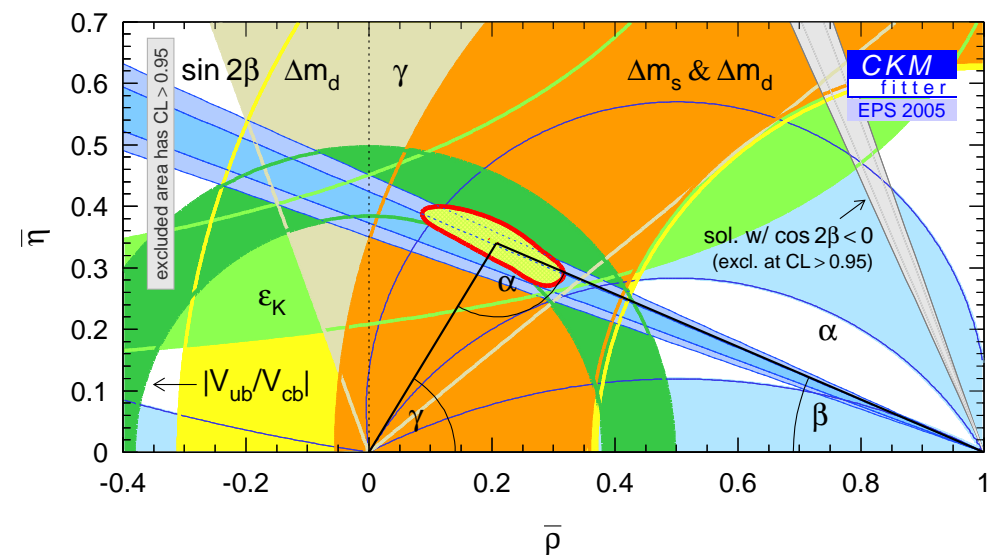

Figure 8. Constraints in the $\rho-\eta$ plane from recent measurements at B-factories.

with existing constraints.

\section{Rare Processes}

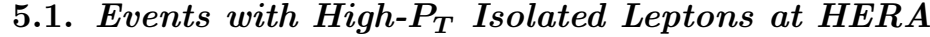

In $e p$ collisions at HERA, the production of a $W$ boson has a cross-section of about $1 \mathrm{pb}$. The leptonic decay of the $W$ leads to events with an isolated lepton (electron, muon or tau) and large missing transverse momentum. Of particular interest are events where also the hadronic system has a large transverse momentum $\left(P_{T}^{X}\right)$. The $\mathrm{H} 1$ experiment observes an abnormally large rate of such events in the electron and muon channels ${ }^{13}$. In the analysis of all HERA-I and HERA-II data, which amount to a total luminosity of $279 \mathrm{pb}^{-1}, 17$ events are observed at $P_{T}^{X}>25 \mathrm{GeV}$, whereas the SM expectation is $9.0 \pm 1.5$. Only 2 of these events are observed in $e^{-} p$ collisions, in agreement with the SM expectation, while 15 events are observed in the $e^{+} p$ data where $4.6 \pm 0.8$ events are expected (see Table 1 ). The ZEUS experiment has carried out a re-analysis of the electron channel using the

Table 1. The number of isolated lepton events with $P_{T}^{X}>25 \mathrm{GeV}$ observed by H1 and ZEUS, compared with the SM predictions.

\begin{tabular}{ccccc}
\multicolumn{1}{c}{$P_{T}^{X}>25 \mathrm{GeV}$} & $\begin{array}{c}\text { Electron } \\
\text { obs/exp }\end{array}$ & $\begin{array}{c}\text { Muon } \\
\text { obs/exp }\end{array}$ & $\begin{array}{c}\text { Combined } \\
\text { obs/exp }\end{array}$ \\
\hline H1 $e^{-} p, 121 \mathrm{pb}^{-1}(98-05)$ & $2 / 2.4 \pm 0.5$ & $0 / 2.0 \pm 0.3$ & $2 / 4.4 \pm 0.7$ \\
ZEUS $e^{-} p, 143 \mathrm{pb}^{-1}(98-05)$ & $3 / 2.9 \pm 0.5$ & - & - \\
\hline H1 $e^{+} p, 158 \mathrm{pb}^{-1}(94-04)$ & $9 / 2.3 \pm 0.4$ & $6 / 2.3 \pm 0.4$ & $15 / 4.6 \pm 0.8$ \\
ZEUS $e^{+} p, 106 \mathrm{pb}^{-1}(99-04)$ & $1 / 1.5 \pm 0.13$ & - & - \\
\hline
\end{tabular}


1998-2005 $e^{-} p$ and 1999-2004 $e^{+} p$ data ${ }^{14}$, resulting in a larger purity of the selected $W$ events. The event numbers found in this analysis are also shown in Table 1. At $P_{T}^{X}>25 \mathrm{GeV}$ the number of events observed by ZEUS is in agreement with the SM expectation. A direct comparison of the results of both experiments remains difficult since $\mathrm{H} 1$ and ZEUS cover different phase-space regions. A recent analysis of the $\tau$ channel with a total luminosity of $278 \mathrm{pb}^{-1}$ was also presented by the $\mathrm{H} 1$ collaboration $^{15}$. In the $\tau$ channel, the separation of the $W$ signal from other SM processes is more difficult and the purity and efficiency are lower than for the $e$ and $\mu$ channels. In total 25 events are observed compared to a SM expectation of $24.2 \pm 4.6$. Three of them have $P_{T}^{X}>25 \mathrm{GeV}$ where $0.74 \pm 0.19$ events are expected. The $P_{T}^{X}$ distribution is presented in Figure 9.

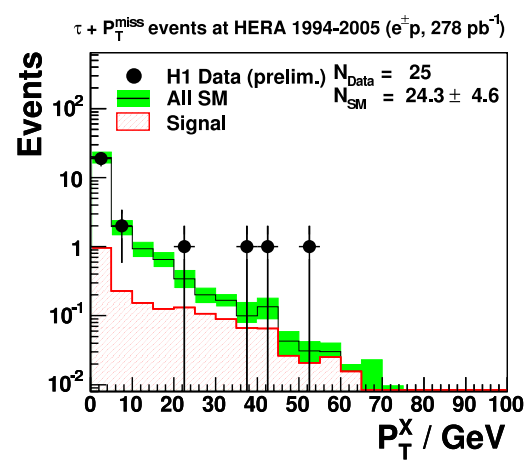

Figure 9. The hadronic transverse momentum distribution of $\tau+P_{T}^{\text {miss }}$ events observed by the $\mathrm{H} 1$ experiment is compared to the SM expectation.

\subsection{Multi-Lepton Events at HERA}

The main production mechanism for multi-lepton events is photon-photon collisions. All event topologies with high $P_{T}$ electrons and muons have been investigated by the $\mathrm{H} 1$ experiment ${ }^{16}$ using a total luminosity of $275 \mathrm{pb}^{-1}$. The measured yields of di-lepton and tri-lepton events are in good agreement with the SM, except in the tail of the distribution of the scalar sum of $P_{T}$ of the leptons $\left(\sum P_{T}\right)$. In $e^{+} p$ collisions, 4 events are observed with $\sum P_{T}>100 \mathrm{GeV}$ compared to a SM prediction of $0.6 \pm 0.1$. No such event is observed in $e^{-} p$ collisions with a similar SM expectation of $0.5 \pm 0.1$. A first measurement of the cross-section of $\tau$-pair production in $e p$ collisions was also presented by $\mathrm{H}^{15}$. 


\section{Searches for New Resonances}

\subsection{New Gauge Bosons}

New resonances coupling to leptons are predicted in several extensions of the SM. Extending the SM gauge group often leads to additional $Z^{\prime}$ bosons, with most popular models being based on left-right symmetry or on the $E_{6}$ Grand Unification group. Both D0 and CDF experiments have looked for di-lepton resonances in the Run II data, including all three channels with dielectrons, di-muons and di-taus. Good agreement with the SM predictions is observed in the di-lepton invariant mass distributions, up to the highest masses ${ }^{17}$. The highest direct bound on $M_{Z^{\prime} \rightarrow e e}>850 \mathrm{GeV}$ is set by CDF using a total luminosity of $820 \mathrm{pb}^{-1}$. The possible production of a $W^{\prime}$ boson decaying into $e-\nu_{e}$ was also investigated by $\mathrm{CDF}^{17}$. No signal is observed and a lower bound on the $W^{\prime}$ mass of $788 \mathrm{GeV}$ was obtained.

\subsection{Leptoquarks}

An intriguing characteristic of the SM is the observed symmetry between the lepton and the quark sectors, which is manifest in the representation of the fermion fields under the SM gauge group, and in their replication over the three family generations. Models which implement a new symmetry between the lepton and quark sectors lead to new "lepto-quark" interactions. Leptoquarks (LQs) are new scalar or vector color-triplet bosons, carrying a fractional electromagnetic charge and both a baryon and a lepton number. Several types of LQs might exist, differing in their quantum numbers. The Buchmüller-Rückl-Wyler (BRW) classification of LQs is based on the assumption that LQs have purely chiral couplings to SM fermions and obey the SM $S U(2)$ symmetry. The interaction of a LQ with a lepton-quark pair is of Yukawa or vector type and is parametrised by a coupling constant $\lambda$. Depending on its quantum numbers, a LQ couples to $e q, \nu q$ or both. The branching ratios $\beta$ for a LQ to decay into $e q$ or $\nu q$ are fixed in the minimal BRW model, but can be treated as a free parameter, e.g. if the LQs mix with other new heavy particles.

At the Tevatron, leptoquarks are mainly pair-produced via their coupling to gluons. First generation LQs have been looked for in Run II data in the eejj, e $\nu j j$ and $\nu \nu j j$ final states ( $j$ denotes a jet). Masses below $256 \mathrm{GeV}$ for LQs decaying solely into an electron and a quark are ruled out by the D0 experiment, combining Run I and Run II data. In comparison, in deep inelastic scattering, LQs are produced through their Yukawa-type coupling $\lambda$. HERA experiments ${ }^{18}$ exclude LQ masses below $\sim 290 \mathrm{GeV}$, if 
the coupling $\lambda$ is of electromagnetic strength $\left(\lambda^{2} / 4 \pi=\alpha_{e m}\right)$. The searches at the Tevatron for scalar LQs with $\beta=0$ in the $\nu \nu j j$ channels have been updated using events with two acoplanar jets and missing transverse energy and new mass bounds of 136 and $117 \mathrm{GeV}$ are set by D0 and CDF, respectively.

Leptoquarks coupling to second or third generation fermions and with masses above $100 \mathrm{GeV}$ can be directly searched for only at the Tevatron. Pair-produced LQs leading to final states with two muons or one muon and missing transverse energy and two jets $(\mu \mu j j$ or $\mu \nu j j)$ have been searched for in the Run II data. For $\beta=1$ and second generation LQs, mass bounds of $224 \mathrm{GeV}$ using Run II data only and $251 \mathrm{GeV}$ by combining Run I and II data are obtained by the CDF and D0 experiments. CDF also looked for third generation LQs in the $\tau \tau j j$ channel ${ }^{17}$ and found lower limits of $344 \mathrm{GeV}$ and $151 \mathrm{GeV}$ on the masses of vector or scalar LQs, respectively.

\subsection{Excited Fermions}

The observed replication of three fermion families motivates the possibility of a new scale of matter yet unobserved. An unambiguous signature for a new scale of matter would be the direct observation of excited states of fermions $\left(f^{*}\right)$, via their decay into a gauge boson. The interaction of an $f^{*}$ with a gauge boson can be effectively described by a magnetic coupling (i.e., a dimension-five operator) proportional to $1 / \Lambda$ where $\Lambda$ is a new scale. Proportionality constants $f, f^{\prime}$ and $f_{s}$ result in different couplings to $U(1)$, $S U(2)$ and $S U(3)$ gauge bosons. Recently, the H1 experiment has carried out a search for excited neutrinos taking advantage of the new sensitivity brought by $114 \mathrm{pb}^{-1}$ of HERA-II data from $e^{-} p$ collisions ${ }^{19}$. The bound on the $\nu^{*}$ mass obtained as a function of $f / \Lambda$ is presented in Figure 10a. Assuming $f / \Lambda=1 / M_{\nu^{*}}$ and $f=-f^{\prime}$, masses below $188 \mathrm{GeV}$ are ruled out. The single production of excited muons $\mu^{*}$ has been looked for at the Tevatron by both CDF and D0 experiments. In an analysis based on a contact interaction formalism for the production and also possibly for the decay of the $\mu^{*}$, lower mass limits of $800 \mathrm{GeV}$ and $618 \mathrm{GeV}$ for $\Lambda=1 \mathrm{TeV}$ can been set by CDF and D0, respectively. Figure 10b presents the corresponding exclusion region for $M_{\mu^{*}}$ as a function of $f / \Lambda$ obtained in the CDF analysis if the same model as the one used by H1 is considered. The D0 experiment also looked for heavy resonances in the $Z+$ jet channel as a signal of excited quark production. Assuming a production and decay mechanism via contact interactions, $q^{*}$ masses below $520 \mathrm{GeV}$ have been 
excluded ${ }^{17}$.

(a)

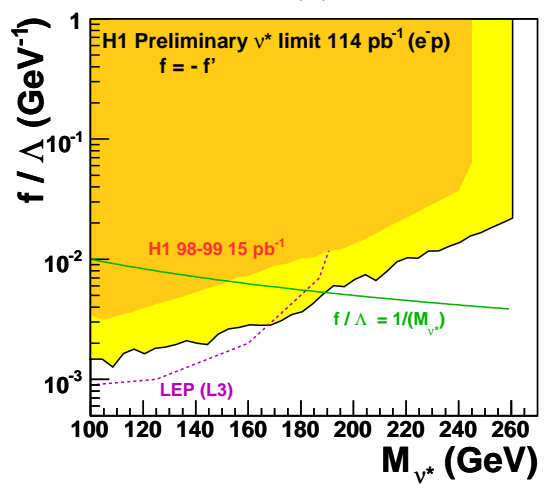

(b)

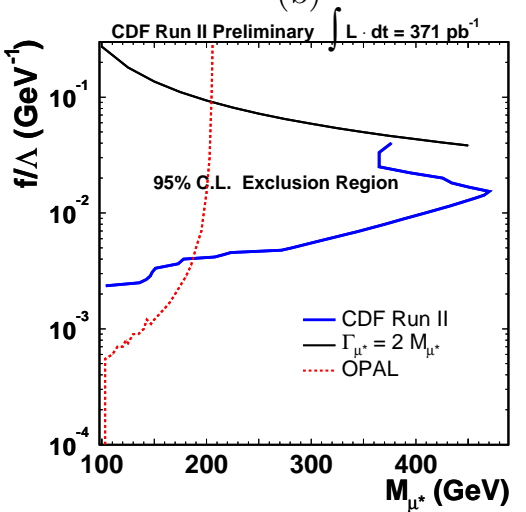

Figure 10. Exclusion limits on the production of excited neutrinos (a) and muons (b) obtained by the $\mathrm{H} 1$ and $\mathrm{CDF}$ experiments, respectively.

\section{Supersymmetry}

A popular extension to the SM is supersymmetry (SUSY). SUSY unifies internal symmetries with Lorentz invariance and associates supersymmetric partners (sparticles) to the known SM particles. Supersymmetric models provide solutions to many problems of the SM (hierarchy, fine-tuning, unification) and predict spectacular final states in high-energy particle collisions. Despite extensive studies at colliders and elsewhere, no unambiguous signal of SUSY has yet been detected. The production of single sparticles is possible if the conservation of the multiplicative quantum number $R_{p}$ is violated $\left(R\right.$-parity is $R_{p}=(-1)^{3 B+L+2 S}$ where $B, L$ and $S$ denote the particle's baryon number, lepton number, and spin, respectively). In $R$-parity violating models, $s$-channel $s$ quark production at HERA via the electronquark-squark Yukawa coupling $(\lambda)$ is possible. A special case is the stop $(\tilde{t})$ which in many SUSY scenarios is the lightest $s$ quark. A recent search for $\tilde{t}$ production at HERA was reported by the ZEUS experiment ${ }^{20}$. The analysis involved both $R_{p}$-violating and gauge decays of the stop and is therefore sensitive to a large class of models, corresponding to a wide scan over the parameter space. The exclusion limits obtained are in agreement with previous results from $\mathrm{H} 1$ and scenarios with stop quarks with mass below $\sim 260 \mathrm{GeV}$ can be excluded if $\lambda^{\prime}=0.3$ (see Figure 11a). 

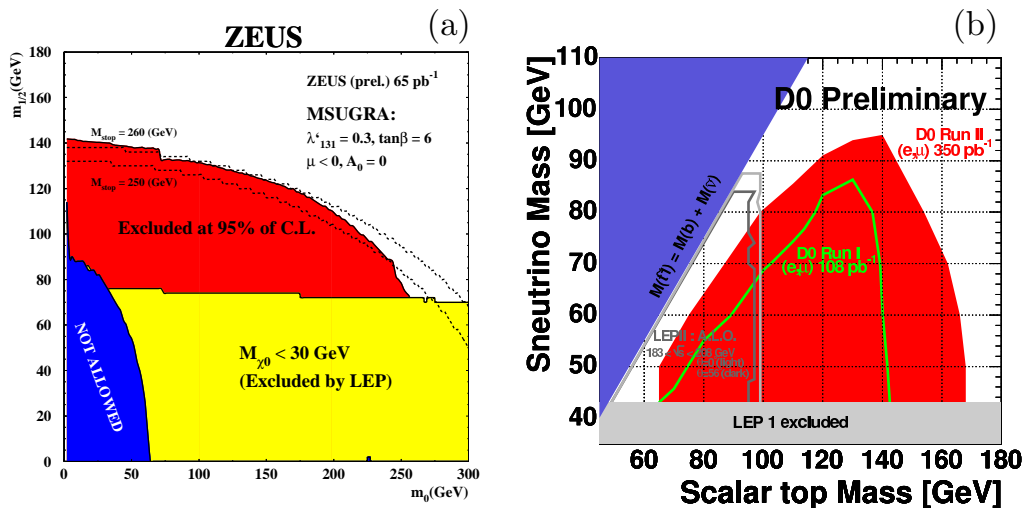

Figure 11. Stop quark production: parameter space regions excluded by ZEUS (a) and D0 (b) in $R_{p}$-violating and $R_{p}$-conserving scenarios, respectively.

Searches where $R_{p}$ is conserved are mainly based on the presence of the lightest supersymmetric particle in the final state, which usually involves large missing transverse momentum. In this case, SUSY particles can only be produced in pairs. A search for the production of stop pairs has been performed by D0, considering scenarios where the lightest squark is a stop $\tilde{t}_{1}$. Final states with $e^{ \pm} \mu^{\mp}+b \bar{b}+E_{T}^{\prime}$ and $\mu^{ \pm} \mu^{\mp}+b \bar{b}+E_{T}^{\prime}$ have been investigated $^{21}$, but no deviations from the SM expectations were observed. The excluded region is presented in Figure 11b. Looking for the possible production of charginos and neutralinos in mSUGRA scenarios where charginos and neutralinos decay leptonically, a large variety of final states with three leptons and missing energy have been investigated by $\mathrm{CDF}^{21}$. Integrated luminosities of $\sim 700 \mathrm{pb}^{-1}$ are used but no significant excess is observed.

If Supersymmetry is realized in Nature, we may expect that experiments at the Tevatron and the LHC will announce its discovery in the near future. For high-precision analyses of supersymmetric parameters with the aim to reconstruct the fundamental supersymmetric theory, one will have to wait for the completion of high-luminosity runs at the LHC and the contribution from experiments at the planned $\mathrm{ILC}^{22}$. Whereas algorithms for SUSY analyses of future experimental results have been worked in great detail, the discovery of non-SUSY new physics will require the development of new strategies $^{23}$. 


\section{Conclusions}

Despite the impressive amount of precision electroweak measurements, no clear sign of deviations from SM expectations has been observed. Nevertheless important discoveries seem to be quite close. The experimental results strongly indicate a light Higgs boson; its possible mass range will be accurately scanned in the next few years both by the Tevatron and LHC experiments hopefully clarifying the status of the SM Higgs sector. The precision measurements of the B-factories, which will double their statistics in the next couple of years, will also contribute in testing the SM by means of $\mathrm{CP}$-violation and rare decays studies.

\section{References}

1. P. Schieferdecker, in these proceedings ${ }^{\mathrm{a}}$.

2. M. P. Giordani, in these proceedings.

3. S. Shimizu, in these proceedings.

4. M. Tomoto, in these proceedings.

5. R. Ranieri, in these proceedings.

6. S. Cabrera, in these proceedings.

7. J. Garcia, in these proceedings.

8. N. Kidonakis, in these proceedings.

9. W. Menges, in these proceedings.

10. Y. Iwasaki, in these proceedings.

11. Y. Kwon, in these proceedings.

12. E. Paoloni, in these proceedings.

13. D. South, in these proceedings.

14. M. Corradi, in these proceedings.

15. S. Xella, in these proceedings.

16. C. Vallée, in these proceedings.

17. J. Kim, in these proceedings.

18. C. Helebrant, in these proceedings.

19. C. Diaconu, in these proceedings.

20. A. Montanari, in these proceedings.

21. F. Badaud, in these proceedings.

22. SPA collaboration, presented by P. Zerwas, in these proceedings.

23. B. Knuteson, in these proceedings.

aProceedings of the XIV International Workshop on Deep Inelastic Scattering, DIS2006, Tsukuba, Japan, 2006, April 20 - 24, see http://www-conf.kek.jp/dis06/ 